\title{
A eficácia dos cuidados preventivos da enfermagem na Pneumonia associada à ventilação mecânica
}

The effectiveness of preventive nursing care in Pneumonia associated with mechanical ventilation

La efectividad de los cuidados de enfermería preventivos en la neumonía asociada a la ventilación mecánica

Recebido: 09/05/2021 | Revisado: 18/05/2021 | Aceito: 26/05/2021 | Publicado: 11/06/2021

Laís Ribeiro Honorato

ORCID: https://orcid.org/0000-0002-4457-9021 Prefeitura Municipal de Volta Redonda, Brasil E-mail:lais.honorato123@gmail.com

André Luiz de Souza Braga

ORCID: https://orcid.org/0000-0002-7961-9038 Universidade Federal Fluminense, Brasil E-mail: andre.braga@globo.com

Deise Ferreira de Souza

ORCID: https://orcid.org/0000-0002-4294-9957 Universidade Federal Fluminense, Brasil E-mail:dfsnit@hotmail.com

Pedro Ruiz Barbosa Nassar

ORCID: https://orcid.org/0000-0002-9238-0519 Universidade Federal Fluminense, Brasil

E-mail: pedrornassar@gmail.com

Suely Lopes de Azevedo

ORCID: https://orcid.org/0000-0003-1107-3427 Universidade Federal Fluminense, Brasil E-mail: suelyazevedo@id.uff.br

\begin{abstract}
Resumo
Objetivo: descrever a eficácia dos cuidados de enfermagem baseados em evidências científicas, disponíveis atualmente na literatura para prevenção de pneumonia associada à ventilação mecânica em pacientes na Unidade de Terapia Intensiva. Método: revisão integrativa da literatura, utilizado as três bases de dados MEDILINE, LILACS e BDENF, incluindo os descritores prevenção e pneumonia associada à ventilação mecânica, cuidados de enfermagem e pneumonia associada à ventilação mecânica e cuidados de enfermagem e prevenção" Resultado: Foram incluídos 15 estudos, encontrados 7 cuidados de enfermagem que contribuem na prevenção da pneumonia associada à ventilação mecânica. A adesão dos profissionais aos cuidados para a prevenção de pneumonia associada à ventilação mecânica deve ser reconhecida, no seu cotidiano, como ações efetivas no processo de trabalho, tendo na educação permanente, o alicerce para garantir a eficácia dos cuidados. Por se tratar de uma mudança eficaz, a educação e o treinamento dos profissionais de saúde são fatores-chave para aumentar a adesão às diretrizes. Nesse contexto, monitorar o feedback da equipe multidisciplinar sobre as medidas para prevenir a PAVM pode ser uma estratégia que aponta lacunas e indicadores de saúde para subsidiar os investimentos na melhoria das práticas de assistência médica, com base no treinamento e na educação permanente. Diante disso, nesta revisão foram identificadas evidências científicas que descrevem a eficácia dos cuidados de enfermagem na prevenção da PAVM, sendo eles garantindo assim, nossa autonomia na gestão do cuidado.
\end{abstract}

Palavras-Chave: Cuidados de enfermagem; Planejamento de assistência ao paciente; Pneumonia associada à ventilação mecânica; Suporte ventilatório interativo; Prevenção.

\begin{abstract}
Objective: to describe the effectiveness of nursing care based on scientific evidence, currently available in the literature for the prevention of pneumonia associated with mechanical ventilation in patients in the Intensive Care Unit. Method: integrative literature review, using the three MEDILINE, LILACS and BDENF databases, including the keywords prevention and pneumonia associated with mechanical ventilation, nursing care and pneumonia associated with mechanical ventilation and nursing care and prevention" Result: Included 15 studies, found 7 nursing care that contribute to the prevention of pneumonia associated with mechanical ventilation. The professionals' adherence to care for the prevention of pneumonia associated with mechanical ventilation must be recognized, in their daily lives, as effective actions in the work process, having in permanent education, the foundation to guarantee the effectiveness of care. As it is an effective change, the education and training of health professionals are key factors to
\end{abstract}


increase adherence to the guidelines. In this context, monitoring the multidisciplinary team's feedback on measures to prevent VAP can be a strategy that points out gaps and health indicators to subsidize investments in improving health care practices, based on training and continuing education. Therefore, in this review, scientific evidence was identified that describes the effectiveness of nursing care in preventing VAP, thus guaranteeing our autonomy in care management.

Keywords: Nursing care; Patient care planning; Pneumonia associated with mechanical ventilation; Interactive ventilatory support; Prevention.

\section{Resumen}

Objetivo: describir la efectividad de los cuidados de enfermería con base en la evidencia científica, actualmente disponible en la literatura para la prevención de la neumonía asociada a la ventilación mecánica en pacientes en la Unidad de Cuidados Intensivos. Método: revisión integradora de la literatura, utilizando las tres bases de datos MEDILINE, LILACS y BDENF, incluyendo las palabras clave prevención y neumonía asociada a ventilación mecánica, cuidados de enfermería y neumonía asociada a ventilación mecánica y cuidados y prevención de enfermería" Resultado: Incluyó 15 estudios, encontró 7 enfermería cuidados que contribuyan a la prevención de la neumonía asociada a la ventilación mecánica. La adherencia de los profesionales a los cuidados para la prevención de la neumonía asociada a la ventilación mecánica debe ser reconocida, en su vida diaria, como acciones efectivas en el proceso de trabajo, teniendo en la educación permanente, la base para garantizar la efectividad del cuidado. Por tratarse de un cambio efectivo, la educación y formación de los profesionales sanitarios son factores clave para incrementar la adherencia a las guías. En este contexto, el seguimiento de la retroalimentación del equipo multidisciplinario sobre las medidas de prevención de NAV puede ser una estrategia que señale brechas e indicadores de salud para subsidiar inversiones en la mejora de las prácticas de atención de salud, basadas en la capacitación y la educación continua. Por tanto, en esta revisión se identificó evidencia científica que describe la efectividad del cuidado de enfermería en la prevención de NAV, garantizando así nuestra autonomía en la gestión del cuidado.

Palabras- clave: Cuidado de enfermera; Planificación de la atención al paciente; Neumonía asociada a ventilación mecánica; Soporte ventilatorio interactivo; Prevención.

\section{Introdução}

A pneumonia hospitalar é definida como aquela que ocorre a partir de 48 horas da admissão do paciente e a segunda principal Infecção Relacionada à Assistência à Saúde (IRAS) em Unidade de Terapia Intensiva (UTI). Frequentemente está associada ao uso da ventilação mecânica (VM), sendo denominada Pneumonia Associada à Ventilação Mecânica (PAVM). Trata-se assim da complicação infecciosa mais prevalente em terapia intensiva, com taxas que variam de $9 \%$ a $40 \%$ das IRAS adquiridas nessa unidade. A PAVM está associada a um aumento no período de hospitalização e descrevem-se índices de mortalidade que podem variar de $24 \%$ a $76 \%$, repercutindo de maneira significativa nos custos hospitalares (SILVA, 2016).

A pneumonia associada à ventilação mecânica (PAVM) é a segunda infecção mais frequente em UTIs americanas e a mais frequente em UTIs européias (Sociedade Paulista De Infectologia, 2017). No Brasil, em que pese à ausência de dados nacionais e multicêntricos, experiências individuais mostram as PAVM como as mais frequentes infecções dentro da UTI. Sua incidência está interligada ao uso inadequado de medidas que previnem o surgimento dessa infecção nas UTI's. A adoção de medidas preventivas de forma adequada garante a minimização da ocorrência desta patologia que ascende com frequência nas UTI's (Honório, 2015).

A maior permanência de contato com o paciente criticamente enfermo fica sob os cuidados da enfermagem. Esta categoria é responsável por realizar a maioria das medidas preventivas referentes à PAVM através do bundle de prevenção, que é um grupo de intervenções baseadas em evidências científicas, que quando adotadas em conjunto resultam em melhores resultados do que adotadas individualmente (Institute For Healthcare Improvement, 2012).

A assistência aos pacientes na UTI está relacionada ao cuidado direto e intensivo, com monitoramento permanente, proporcionando uma assistência humanizada, de qualidade e livre de riscos, onde requer qualificação profissional e conhecimentos específicos, devido ao perfil e gravidade dos pacientes. Justifica-se, portanto, a necessidade da capacitação permanente da equipe multiprofissional, incluindo a equipe de enfermagem, presente 24 horas no cuidado ao paciente crítico. 
Segundo Inoue e Matsuda (2009), a UTI implica em elevada carga de trabalho devido à alocação de pacientes sujeitos às constantes alterações hemodinâmicas e iminente risco de morte, os quais exigem cuidados complexos, atenção ininterrupta $\mathrm{e}$ tomada de decisão imediata. Nesse sentido, Gomes (1988), afirma que o enfermeiro que atua na UTI necessita de conhecimento cientifico, prática e técnica, a fim de que possa tomar decisões rápidas e concretas, transmitindo segurança a toda equipe e diminuindo os riscos que ameaçam a vida do paciente.

Nesse contexto, este estudo tem como objetivo descrever a eficácia dos cuidados de enfermagem baseados em evidências científicas, disponíveis atualmente na literatura para prevenção de pneumonia associada à ventilação mecânica em pacientes na Unidade de Terapia Intensiva.

\section{Metodologia}

Estudo do tipo revisão integrativa. Este método possibilita inclui a análise de pesquisas relevantes que dão suporte para a tomada de decisão e a melhoria da prática clínica, possibilitando a síntese do estado do conhecimento de um determinado assunto, além de apontar lacunas do conhecimento que precisam ser preenchidas com a realização de novos estudos. Para tanto, foram adotadas as seis etapas indicadas para a constituição da revisão integrativa da literatura: Primeira etapa: identificação do tema e seleção da hipótese ou questão de pesquisa para a elaboração da revisão integrativa; Segunda etapa: estabelecimento de critérios para inclusão e exclusão de estudos/ amostragem ou busca na literatura; Terceira etapa: definição das informações a serem extraídas dos estudos selecionados/ categorização dos estudos; Quarta etapa: avaliação dos estudos incluídos na revisão integrativa; Quinta etapa: interpretação dos resultados; Sexta etapa: apresentação da revisão/síntese do conhecimento. (Mendes, 2008).

A seleção foi realizada a partir do levantamento de trabalhos indexados nas bases: MEDLINE, LILACS, BDENF, acessado através da Biblioteca Virtual em Saúde (BVS). Foram utilizados os descritores: pneumonia associada à ventilação mecânica/ prevenção / cuidados de enfermagem.

Desta forma o levantamento foi realizado em outubro de 2019. Foram incluídos os artigos publicados no período de 2015 a 2019.

A questão norteadora considerada foi: Quais são as evidências científicas encontradas na literatura para os cuidados de enfermagem na prevenção da pneumonia associada à ventilação mecânica?

Foram excluídos os artigos que não abordaram especificamente os cuidados de enfermagem na prevenção da pneumonia associada à ventilação mecânica.

A análise e a interpretação dos dados foram por meio da leitura exploratória dos estudos cuja finalidade catalogou-se em averiguar a relevância do material científico.

Realizou-se, na sequência, a leitura seletiva para a composição de uma estrutura teórica que respondesse à pergunta norteadora e ao objetivo do estudo. Concluiu-se tal processo na seleção dos artigos descritos neste estudo.

A prática baseada em evidências preconiza sistemas de classificação, dependendo da abordagem metodológica adotada, que toma por base o delineamento do estudo (Oxford Centre For Evidence-Based Medicine, 2011).

\section{Resultados}

A amostra compreendeu 14 estudos, selecionados conforme demonstra o fluxograma de seleções publicadas (Figura 1). 
Figura 1 - Fluxograma de seleção dos artigos analisados. Niterói (RJ), Brasil, 2020.

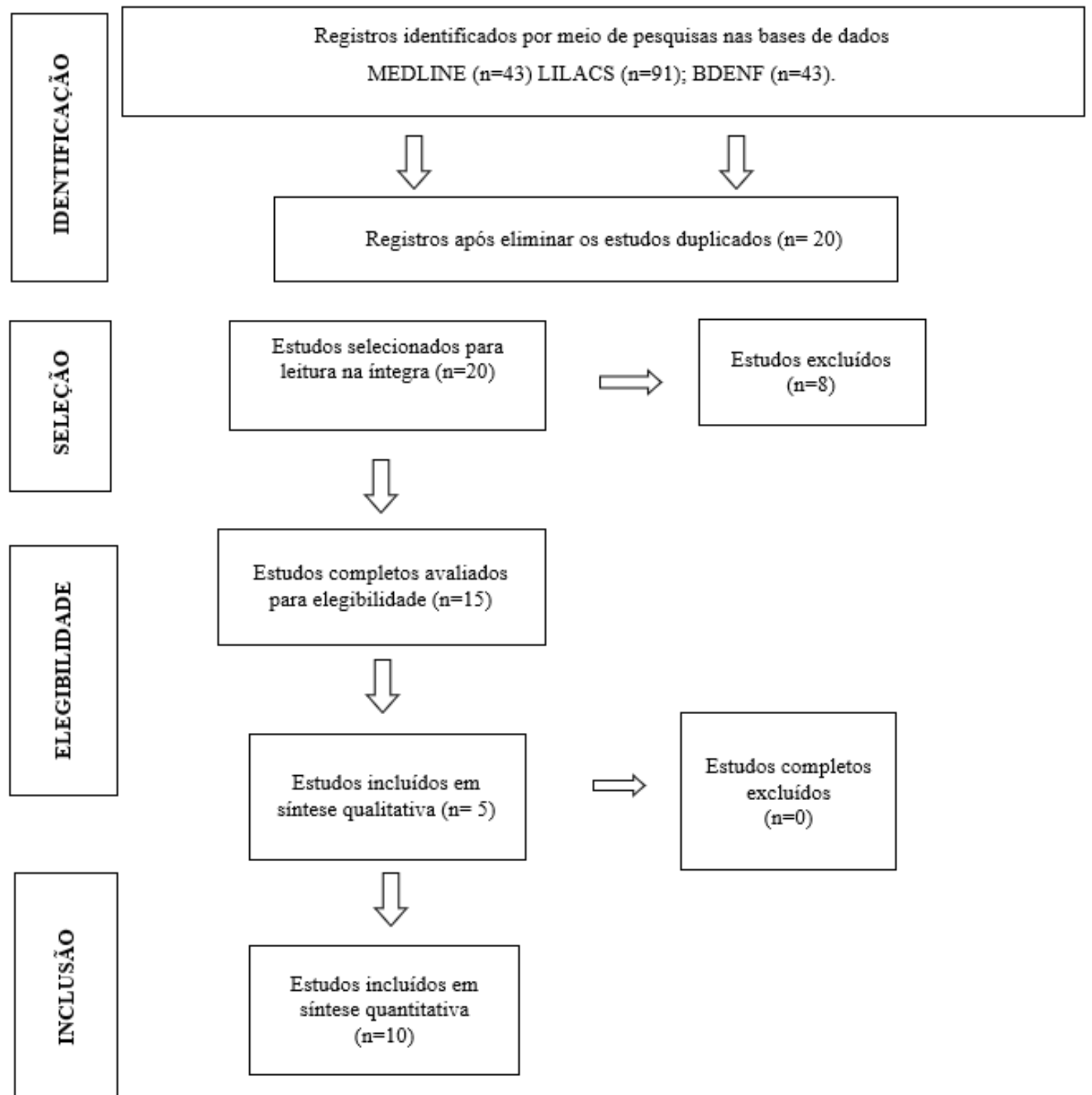

Fonte: Autores.

Identificou-se um total de 177 artigos, sendo 91 na LILACS, 43 na BDENF, 43 na MEDLINE/PubMed. Destes, 143 foram excluídos por não se referirem à temática em estudo ou não responderiam à questão norteadora. Encontrados 20 duplicidades de artigos. Assim, foram selecionados 14 artigos que constituíram essa revisão integrativa (MENDES, 2008).

Dos quatorze artigos selecionados de acordo com a temática dessa pesquisa, um foi publicado ano de 2015, três no ano de 2016, um de 2017 e nove publicados no ano de 2019.

No que se refere ao delineamento do estudo a pesquisa Descritiva-quantitativa foi base para sete publicações, tendo nível de evidência 4.

Com a finalidade de facilitar a exposição dos estudos incluídos nesta revisão, os mesmos foram agrupados na Tabela 1, a partir do título, ano de publicação, delineamento e nível de evidência científica, ordenadamente em sequência decrescente, dos estudos publicados de 2015 a 2019. 
Research, Society and Development, v. 10, n. 7, e0610715935, 2021

(CC BY 4.0) | ISSN 2525-3409 | DOI: http://dx.doi.org/10.33448/rsd-v10i7.15935

Tabela 1: Caracterização dos artigos. Niterói, 2020.

\begin{tabular}{|c|c|c|c|c|c|c|}
\hline Autores & Ano & Tipo de pesquisa & Título & Periódico & Base & Nível de Evidência \\
\hline ALMEIDA, K.M. $V$ et al. & 2015 & Descritivo-Quantitativo & $\begin{array}{l}\text { Adesão às medidas de } \\
\text { prevenção para pneumonia } \\
\text { agsociada à ventilação } \\
\text { mecânica }\end{array}$ & $\begin{array}{c}\text { Revista de Enfermagem da } \\
\text { UFSM }\end{array}$ & $\mathrm{BDENF}$ & 4 \\
\hline Z.ANEI, S.S.V.; et al. & 2016 & Descritivo-Quantitativo & $\begin{array}{l}\text { Valoração e registros sobre a } \\
\text { higiene oral de pacientes } \\
\text { intubados nas unidades de } \\
\text { terapia intensiva }\end{array}$ & $\begin{array}{l}\text { Revista Mineira de } \\
\text { Enfermagem }\end{array}$ & LILACS & 4 \\
\hline BALBNO, C.M; BRAZ, RM; et al. & 2016 & Descritivo-Quantitativo & $\begin{array}{l}\text { Avaliação da técnica de } \\
\text { aspiração de paciente em } \\
\text { ventilação mecânica } \\
\text { realizada pela enfermagem }\end{array}$ & $\begin{array}{l}\text { Revista de Enfermagem } \\
\text { UFPE on line }\end{array}$ & BDENF & 4 \\
\hline RODRIGUES, A.N.; et al. & 2016 & Descritivo-Quantitativo & $\begin{array}{l}\text { Impactos e fatores } \\
\text { determinantes no bundle de } \\
\text { pneumonia associada à } \\
\text { ventilação mecânica }\end{array}$ & $\begin{array}{l}\text { Revista Brasileira de } \\
\text { Enfermagem }\end{array}$ & LILACS & 4 \\
\hline MANSANO, F.P.N.; et al. & 2017 & Quase experimental & $\begin{array}{l}\text { Impacto de ação educativa } \\
\text { na manutenção do decúbito } \\
\text { elevado como medida } \\
\text { preventiva de pneumonia } \\
\text { associada à ventilação } \\
\text { mecânica em Unidade de } \\
\text { Terapia Intensiva }\end{array}$ & $\begin{array}{l}\text { Arquivos Brasileiros de } \\
\text { Ciências da Saúde }\end{array}$ & LILACS & 3 \\
\hline ZIGART, J_A.A.; et al & 2019 & Descritivo-Quantitativo & $\begin{array}{l}\text { Adesão ao protocolo de } \\
\text { pneumonia associado à } \\
\text { ventilação mecânica }\end{array}$ & $\begin{array}{l}\text { Revista de Enfermagem } \\
\text { UFPE on line }\end{array}$ & BDENF & 4 \\
\hline TAMINATO, M; ALECRIM, RC.; et al. & 2019 & Coorte- prospectivo & $\begin{array}{l}\text { Boas práticas na prevenção } \\
\text { de pneumonia agsociada à } \\
\text { ventilação mecânica }\end{array}$ & $\begin{array}{l}\text { Acta Paulista de } \\
\text { Enfermagem }\end{array}$ & LILACS & 3 \\
\hline BARROS, F.M.M & 2019 & Descritivo-prospectivo & $\begin{array}{l}\text { Adesão ao bundle de } \\
\text { prevenção de } \\
\text { associada } \\
\text { mecânica }\end{array}$ & Revista Cuvidarte. & LILACS & 3 \\
\hline
\end{tabular}


Research, Society and Development, v. 10, n. 7, e0610715935, 2021

(CC BY 4.0) | ISSN 2525-3409 | DOI: http://dx.doi.org/10.33448/rsd-v10i7.15935

\begin{tabular}{|c|c|c|c|c|c|c|}
\hline CRUZ, J. R.M; MARTINS, MD.S. & 2019 & Descritivo-longitudinal & $\begin{array}{l}\text { Pneumonia associada à } \\
\text { ventilação } \\
\text { invasiva: mecânica } \\
\text { enfermagem }\end{array}$ & $\begin{array}{c}\text { Revista de Enfermagem } \\
\text { Referência }\end{array}$ & LILACS & 4 \\
\hline LOURENÇONE, E.M.S.; et al. & 2019 & Observacional- longitudinal & $\begin{array}{l}\text { Adesão às medidas } \\
\text { preventivas versus incidência } \\
\text { de pneumonia agsociada à } \\
\text { ventilação mecânica }\end{array}$ & $\begin{array}{l}\text { Revista de Epidemiologia } \\
\text { e Controle de Infecção }\end{array}$ & LILACS & 3 \\
\hline COELHO, A.F.P.; et al. & 2019 & Quasgi- experimental & $\begin{array}{l}\text { O impacto da aprendizagem } \\
\text { interprofissional na } \\
\text { pneumonia agsociada à } \\
\text { ventilação: implementação } \\
\text { de bugndles em uma unidade } \\
\text { de cuidados intenaivos }\end{array}$ & $\begin{array}{l}\text { Revista Oficial do } \\
\text { Conselho Federal de } \\
\text { Enfermagem }\end{array}$ & LILACS & 3 \\
\hline MARAN, E.; SLiGOLORN, D.N.; et al. & 2019 & Descritivo-Quantitativo & $\begin{array}{l}\text { Prevenção da pneumonia } \\
\text { agsociada dà ventilação } \\
\text { mecânica sob a ótica de } \\
\text { acadêmicos de enfermagem }\end{array}$ & $\begin{array}{l}\text { Revista online de pesquisa } \\
\text { cuidado é fundamental }\end{array}$ & LILACS & 4 \\
\hline MELO, M.M., et al, 2019. & 2019 & Transversal quanti-qualitativo & $\begin{array}{l}\text { Pneumonia Associada à } \\
\text { Ventilação } r \text { Mecânica: } \\
\text { Conhecimento ros } \\
\text { Profissionais de Saúde } \\
\text { Acerca da Prevenção e } \\
\text { Medidas Educativas }\end{array}$ & $\begin{array}{l}\text { Revista online de pesquisa } \\
\text { cuidado é fundamental }\end{array}$ & LILACS & 3 \\
\hline DUTRA, L_A.; et al. & 2019 & $\begin{array}{l}\text { Descritivo-Qualitativo- } \\
\text { Exploratório }\end{array}$ & $\begin{array}{l}\text { Pneumonia associada à } \\
\text { ventilação mecânica: } \\
\text { percepção dos profissionais } \\
\text { de enfermagem }\end{array}$ & $\begin{array}{l}\text { Revista de Enfermagem } \\
\text { UFPE on line }\end{array}$ & BEDENF & 4 \\
\hline
\end{tabular}


Após a leitura, análise e síntese do conteúdo dos artigos, foram identificados os cuidados de enfermagem eficazes literatura para prevenção de pneumonia associada à ventilação mecânica em pacientes na Unidade de Terapia Intensiva. (Quadro 1).

Quadro 1 - Artigos selecionados da pesquisa.

\begin{tabular}{|c|c|c|c|}
\hline Cuidados de enfermagem & Procedimentos de enfermagem & Estudos & Evidências \\
\hline \multirow[t]{3}{*}{$\begin{array}{l}\text { Higiene oral e das mãos na } \\
\text { prevenção da PAVM }\end{array}$} & $\begin{array}{l}\text { Realizar higienização rigorosa das mãos, } \\
\text { independente do uso de luvas. }\end{array}$ & $\begin{array}{l}\text { Pneumonia associada à ventilação } \\
\text { mecânica invasiva: cuidados de } \\
\text { enfermagem }\end{array}$ & $\begin{array}{l}\text { A frequência de pneumonia nos doentes } \\
\text { ventilados foi baixa, verificando-se uma } \\
\text { elevada taxa de adesão à bundle. }\end{array}$ \\
\hline & & CRUZ, J.M.R.; et al, 2019. & \\
\hline & $\begin{array}{l}\text { Realizar higiene oral com Gluconato de } \\
\text { Clorexidina } 0,12 \%\end{array}$ & $\begin{array}{l}\text { Adesão ao bundle de prevenção de } \\
\text { pneumonia associada à ventilação } \\
\text { mecânica } \\
\text { BARROS, F.R.B.; et al, } 2019 \text {. }\end{array}$ & $\begin{array}{l}\text { Apesar da baixa adesão por parte da equipe de } \\
\text { algumas estratégias, as taxas de densidade de } \\
\text { incidência de PAVM por } 1.000 \text { pacientes/dia } \\
\text { diminuíram de } 13,3 \text { na primeira fase para } 11,9 \\
\text { na última. }\end{array}$ \\
\hline \multirow[t]{2}{*}{$\begin{array}{l}\text { A prevenção da broncoaspiração } \\
\text { de secreções }\end{array}$} & $\begin{array}{l}\text { Manter cabeceira elevada }\left(30-45^{\circ}\right) \text {, se } \\
\text { não houver } \quad \text { contraindicação, } \\
\text { principalmente quando receber nutrição } \\
\text { por sonda. }\end{array}$ & $\begin{array}{l}\text { Adesão ao protocolo de pneumonia } \\
\text { associado à ventilação mecânica } \\
\text { ZIGART, J.A.A. et al, } 2019 \text {. }\end{array}$ & $\begin{array}{l}\text { Evidenciou-se, também, menor taxa de PAVM, } \\
\text { quando comparada com outros estudos, } \\
\text { demonstrando a melhoria desse indicador em } \\
\text { UTI a partir das ações preventivas. }\end{array}$ \\
\hline & $\begin{array}{l}\text { Realizar controle efetivo da pressão do } \\
\text { cuff do tubo orotraqueal ou } \\
\text { traqueostomia; manter entre } 20 \text { a } 30 \mathrm{~cm} \\
\mathrm{H} 2 \mathrm{O} \text {. }\end{array}$ & $\begin{array}{l}\text { Adesão às medidas preventivas } \\
\text { versus incidência de pneumonia } \\
\text { associada à ventilação mecânica } \\
\text { LOURENÇONE, E.M.S.; et al, } \\
2019 \text {. }\end{array}$ & $\begin{array}{l}\text { A média da taxa de adesão das medidas } \\
\text { preventivas em } 1.296 \text { avaliações realizadas } \\
\text { evidenciou adequação em: } 94 \% \text { posição do } \\
\text { filtro; } 88,7 \% \text { cabeceira elevada; } 77,3 \% \text { higiene } \\
\text { oral com clorexidina } 0,12 \% \text {; e } 91,7 \% \text { controle } \\
\text { da pressão do cuff. A taxa de densidade de } \\
\text { incidência de PAVM na UTI deste estudo foi } \\
\text { menor em relação às das UTIs. }\end{array}$ \\
\hline \multirow[t]{4}{*}{$\begin{array}{l}\text { Cuidados com a aspiração das } \\
\text { secreções e circuito ventilatório }\end{array}$} & $\begin{array}{l}\text { Realizar aspiração das vias aéreas } \\
\text { somente quando necessário, com } \\
\text { ausculta pulmonar prévia e evitar instilar } \\
\text { fisiológica } 0,9 \% \text { ou de qualquer outra } \\
\text { natureza. }\end{array}$ & \multirow[t]{2}{*}{$\begin{array}{l}\text { Boas práticas na prevenção de } \\
\text { pneumonia associada à ventilação } \\
\text { mecânica } \\
\text { ALECRIN, R.X.; et al, } 2019 .\end{array}$} & \multirow[t]{2}{*}{$\begin{array}{l}\text { O estudo mostrou que quanto maior a adesão às } \\
\text { medidas de boas práticas, menor é o risco de } \\
\text { Pneumonia Associada à Ventilação Mecânica, } \\
\text { porém não foi um dado estatisticamente } \\
\text { significante. }\end{array}$} \\
\hline & $\begin{array}{l}\text { Ter todo cuidado para não fazer nenhuma } \\
\text { contaminação nesse momento. }\end{array}$ & & \\
\hline & $\begin{array}{l}\text { Preferir sistema fechado e/ou aberto de } \\
\text { aspiração para prevenção da PAVM. }\end{array}$ & $\begin{array}{l}\text { Pneumonia associada à ventilação } \\
\text { mecânica invasiva: cuidados de } \\
\text { enfermagem } \\
\text { CRUZ, J.M.R.; et al, } 2019 \text {. }\end{array}$ & $\begin{array}{l}\text { A frequência de pneumonia nos doentes } \\
\text { ventilados foi baixa, verificando-se uma } \\
\text { elevada taxa de adesão à bundle de prevenção à } \\
\text { pneumonia associada à ventilação mecânica. }\end{array}$ \\
\hline & $\begin{array}{l}\text { Manter o circuito do ventilador livre do } \\
\text { acúmulo de água ou condensações. } \\
\text { Quando essas estiverem presentes, } \\
\text { devem ser descartadas. }\end{array}$ & $\begin{array}{l}\text { Pneumonia associada à ventilação } \\
\text { mecânica invasiva: cuidados de } \\
\text { enfermagem } \\
\text { CRUZ, J.M.R.; et al, } 2019 \text {. }\end{array}$ & $\begin{array}{l}\text { A frequência de pneumonia nos doentes } \\
\text { ventilados foi baixa, verificando-se uma } \\
\text { elevada taxa de adesão à bundle de prevenção à } \\
\text { pneumonia associada à ventilação mecânica. }\end{array}$ \\
\hline \multirow{2}{*}{$\begin{array}{c}\text { Avaliação diária da possibilidade } \\
\text { de extubação }\end{array}$} & Evitar sedações desnecessárias. & \multirow{2}{*}{$\begin{array}{l}\text { Boas práticas na prevenção de } \\
\text { pneumonia associada à ventilação } \\
\text { mecânica. } \\
\text { BELASCO, A.G.S.; et al, } 2019 \text {. }\end{array}$} & \multirow{2}{*}{$\begin{array}{l}\text { O estudo mostrou que quanto maior a adesão às } \\
\text { medidas de boas práticas, menor é o risco de } \\
\text { Pneumonia Associada à Ventilação Mecânica. }\end{array}$} \\
\hline & $\begin{array}{l}\text { Prever e antecipar o desmame } \\
\text { ventilatório e extubação. }\end{array}$ & & \\
\hline Posicionamento do filtro & $\begin{array}{l}\text { Posicionar o filtro acima da inserção do } \\
\text { tubo. }\end{array}$ & $\begin{array}{l}\text { Adesão às medidas preventivas } \\
\text { versus incidência de pneumonia } \\
\text { associada à ventilação mecânica } \\
\text { LOURENÇONE, E.M.S.; et al, } \\
2019 \text {. }\end{array}$ & $\begin{array}{l}\text { Com monitorização e reforço contínuo, as } \\
\text { medidas preventivas para PAVM, realizadas } \\
\text { pela equipe de enfermagem, mantiveram-se } \\
\text { acima dos } 77 \% \text {, com a concomitante } \\
\text { diminuição na taxa de PAVM. }\end{array}$ \\
\hline Educação continuada da Equipe & $\begin{array}{l}\text { Realizar } \\
\text { permanente/continuada da equipe sobre } \\
\text { todos os cuidados que envolvem a } \\
\text { prevenção da PAVM e de outras } \\
\text { infecções. }\end{array}$ & $\begin{array}{l}\text { Pneumonia Associada à Ventilação } \\
\text { Mecânica: Conhecimento dos } \\
\text { Profissionais de Saúde Acerca da } \\
\text { Prevenção e Medidas Educativas. } \\
\text { MELO, M.M.; et al, } 2019 \text {. }\end{array}$ & $\begin{array}{l}\text { Evidenciou-se que existe uma fragilidade no } \\
\text { conhecimento dos profissionais relativo à } \\
\text { prevenção de PAVM e o entusiasmo dos } \\
\text { participantes em capacitar-se e contribuir na } \\
\text { implementação de intervenções que visem a } \\
\text { redução das taxas de pneumonia associada à } \\
\text { ventilação mecânica. }\end{array}$ \\
\hline
\end{tabular}




\section{Discussão}

Atualmente, nas UTIs, a infecção pulmonar é uma ameaça aos pacientes submetidos à ventilação mecânica invasiva, que aumenta de forma considerável a morbimortalidade e os custos da assistência intensiva (Zanei et al, 2016).

Os estudos selecionados para esse artigo demostraram vários métodos de prevenção associada à ventilação mecânica como: higienização das mãos, cabeceira elevada de 30 a $40^{\circ}$, higiene bucal com clorexidina à $0,12 \%$, pressão do cuff do tubo endotraqueal entre 20 a $30 \mathrm{~cm} \mathrm{H2O}$, técnica correta de aspiração endotraqueal, interrupção da nutrição enteral ao abaixar a cabeceira, avaliação da pausa diária de sedação e possibilidade de extubação, posicionamento do filtro bacteriológico e educação continuada da equipe.

A maioria das intervenções específicas para a prevenção de PAVM envolvem diretamente a atuação do enfermeiro, quer seja na implementação, na monitorização ou no gerenciamento, o que torna a equipe de enfermagem fundamental para a implementação de estratégias preventivas e educativas. Nesse contexto, a higienização das mãos é considerada o cuidado mais importante e eficaz na prevenção e transmissão das IACS (Cruz et al, 2019).

A higiene oral com uso de antis-sépticos, tal como clorexidina, está associada a baixo risco de desenvolvimento de PAVM, conforme o bundle do Institute for Healthcare Improvement - IHI é recomendado a limpeza pelo menos duas vezes ao dia utilizando clorexidina à $0,12 \%$ (IHI, 2014). Uma revisão com ensaios clínicos randomizados descobriu que enxaguatório bucal ou gel reduziu o risco de PAVM em comparação com o placebo de $25 \%$ para cerca de $19 \%$ (p < 0,05). Além disso, não houve evidência significativa $(\mathrm{p}<0,05)$ entre o uso de gluconato de clorexidina na higiene bucal versus a duração da VM mecânica e a permanência na UTI (Wang, 2016).

A broncoaspiração é um dos fatores que causam PAVM. Estudos sugerem que o posicionamento do decúbito do leito elevado, entre 30 e 45 graus, promove a redução de refluxo e da aspiração de secreções nasofaríngeas, orofaríngeas e gastrointestinais, favorecendo maior volume corrente, com a melhora dos parâmetros ventilatórios com a pressão de suporte, e diminuindo o risco de PAVM em relação a posições inferiores a 30 graus (Zigart et al, 2019).

A manutenção adequada da pressão do cuff nos pacientes submetidos à ventilação mecânica é importante para prevenir microaspirações. O IHI recomenda que pressão permaneça entre 20 e $25 \mathrm{mmHg}$. Um estudo observacional prospectivo demonstrou que esse critério reduziu significativamente a incidência de PAVM (p < 0,05) (Lorente et al, 2014). Por outro lado, uma meta análise mostrou que o controle contínuo da pressão do cuff não teve impacto significativo nos desfechos secundários, como duração e dias livres de VM, tratamento antimicrobiano ou permanência ou mortalidade na UTI $(\mathrm{p}>0,05)$ (Rouzé et al, 2017).

A aspiração de secreções das vias respiratórias de pacientes em ventilação mecânica deve ser realizada quando o paciente apresentar: ruído no tubo traqueal, evidências de ruídos por secreção (durante a ausculta), secreção visível e redução de saturação percutânea (Silva, 2005). Em relação ao tipo de sistema, não existe diferença na incidência de pneumonia associada à ventilação mecânica quando comparados aos sistemas de sucção aberto ou fechado (ANVISA, 2017). O sistema aberto requer a desconexão do paciente do ventilador mecânico e exige técnica asséptica e materiais estéreis. Já o sistema de aspiração fechado não exige a desconexão do circuito do ventilador e envolve um cateter de múltiplo uso envolto por uma cobertura plástica e transparente, flexível e estéril, com vistas a prevenir a contaminação.

Em relação ao circuito ventilatório, Hinrichsen (2004) ressalta que as secreções depositadas nos circuitos ou a ineficiente manutenção dos mesmos podem levar a que as mesmas sejam aerolizadas para dentro da árvore traqueobrônquica do doente ou conspurcar as mãos do profissional, favorecendo a contaminação cruzada. Recomenda-se a troca do circuito em caso de contaminação por fluidos, secreções ou quando estiverem disfuncionantes. Alecrim et al. (2019), propôs em um estudo de coorte prospectivo, um conjunto de boas práticas na prevenção de PAVM, um dos itens foi a troca do circuito a cada 7 dias, porém não houve associação estatisticamente significante entre a ocorrência de PAVM. 
Os pacientes mecanicamente ventilados recebem infusão contínua de sedação para aliviar o desconforto e a dor. Essa administração contínua de sedativos é um preditor independente para maior duração da ventilação mecânica invasiva, maior permanência na UTI e no hospital, portanto, maiores chances de adquirir PAVM (Taminato et al, 2019). A interrupção diária da sedação, assim como a avaliação da possibilidade de extubação dos pacientes, tem sido altamente recomendada para prevenção da PAVM, considerando que pacientes acordados podem contribuir no desmame ventilatório (Almeida et al, 2015). Um estudo sobre protocolo de sedação e interrupção diária trás em seus resultados que as principais razões que influenciam a baixa adesão a interrupção diária da sedação são a falta de aceitação da enfermagem, preocupação com o risco de remoção dos dispositivos pelo paciente e indução de outros comprometimentos respiratórios ou de desconforto para o paciente (Tanios, 2014). Cabe ao enfermeiro a avaliação do paciente juntamente com a equipe técnica de enfermagem, atuando com objetividade em suas tarefas de orientar, interagir e avaliar, sendo o seu papel essencial na prevenção da PAVM.

Quanto ao posicionamento do filtro de barreira, este cuidado não foi muito abordado nos artigos selecionados, mas Lourençone et al. (2019) estudou a adesão à medidas preventivas versus incidência de pneumonia associada à ventilação mecânica, uma das medidas era o posicionamento do filtro posicionado acima da inserção do tubo (boca ou traqueostomia) e sem presença de sujidade. Essa foi à medida preventiva que nos resultados apresentou melhor adesão da equipe de enfermagem concomitante com a diminuição na taxa de PAVM.

A adesão dos profissionais aos cuidados para a prevenção de pneumonia associada à ventilação mecânica deve ser reconhecida, no seu cotidiano, como ações efetivas no processo de trabalho, tendo na educação permanente, o alicerce para garantir a eficácia dos cuidados. Por se tratar de uma mudança eficaz, a educação e o treinamento dos profissionais de saúde são fatores-chave para aumentar a adesão às diretrizes.

Nesse contexto, monitorar o feedback da equipe multidisciplinar sobre as medidas para prevenir a PAVM pode ser uma estratégia que aponta lacunas e indicadores de saúde para subsidiar os investimentos na melhoria das práticas de assistência médica, com base no treinamento e na educação permanente (Coelho et al, 2019).

\section{Considerações finais}

A ventilação mecânica invasiva é uma tecnologia de suporte à vida utilizado em pacientes críticos, pois substituiu a respiração espontânea do paciente, porém pode acarretar em repercussões graves impactando nas taxas de morbimortalidade, em tempo prolongado de ventilação mecânica, no tempo de permanência e no aumento dos custos assistenciais.

A maioria das intervenções específicas para prevenção de PAVM envolve diretamente a atuação do enfermeiro, seja na elaboração, execução ou supervisão, tornando a equipe de enfermagem e seus cuidados fundamentais para implantação das estratégias preventivas.

Diante disso, nesta revisão foram identificadas evidências científicas que descrevem a eficácia dos cuidados de enfermagem na prevenção da PAVM, sendo eles garantindo assim, nossa autonomia na gestão do cuidado.

Espera-se que a descrição da eficácia dos cuidados preventivos da enfermagem obtidos através dessa revisão integrativa possa auxiliar na tomada de decisões e sensibilizar não só a equipe de enfermagem, mas toda a equipe multiprofissional acerca da importância de ações para prevenção da PAVM.

\section{Referências}

Alecrim, R. X., Taminato, M., Belasco, A., Longo, M. C. B., Kusahara, D. M., \& Fram, D. (2019). Strategies for preventing ventilator-associated pneumonia: an integrative review. Revista Brasileira de Enfermagem, 72(2):521-30.

Almeida, K. Barros, O. Santos, G. Valença, M. Cavalcanti, A., \& Ferreira, K. (2015). Adesão às medidas de prevenção para pneumonia associada à ventilação mecânica. Revista de Enfermagem da UFSM, 5(2), 247 - 256. 
Research, Society and Development, v. 10, n. 7, e0610715935, 2021

(CC BY 4.0) | ISSN 2525-3409 | DOI: http://dx.doi.org/10.33448/rsd-v10i7.15935

Balbino, C. M., \& Braz, R. M. et al. (2016). Avaliação da técnica de aspiração de paciente em ventilação mecânica realizada pela enfermagem. Revista de Enfermagem UFPE on line, 10(6): 4797-4803.

Barros, F. M. M (2019). Adesão ao bundle de prevenção de pneumonia associada à ventilação mecânica. Revista Cuidarte, 10(2): e 746.

Brasil. (2017). Agência Nacional de Vigilância Sanitária. Medidas de Prevenção de Infecção Relacionada à Assistência à Saúde. Anvisa.

Centre For Evidence Based Medicine. (2011). Oxford centre for evidence-based medicine 2011 levels of evidence.

Coelho, A. P., et al. (2017). O impacto da aprendizagem interprofissional na pneumonia associada à ventilação: implementação de bundles em uma unidade de cuidados intensivo. Revista do Conselho Federal de Enfermagem, 10(4): 93-100.

Cruz, J. R. M. da, et al (2019). Pneumonia associada à ventilação mecânica invasiva: cuidados de enfermagem. Revista de Enfermagem Referência, ser IV (20), 87-96

Dutra, L. A.; et al. (2019). Pneumonia associada à ventilação mecânica: percepção dos profissionais de enfermagem. Revista de Enfermagem UFPE on line, 13(4): $884-892$

Gomes, A. M. (2019). Enfermagem na unidade de terapia intensiva, (2a ed.).

Hinrichsen, S. L (2004). Biossegurança e controle de infecções: Risco sanitário hospitalar. Medsi.

Honório, F. G. (2015). Cuidados de enfermagem na prevenção da pneumonia associada à ventilação mecânica. https://www.ccih.med.br/cuidados-deenfermagem-na-prevencao-da-pneumonia-associada-a-ventilacao-mecanica/.

Inoue, K. C. \& Matsuda, L. M (2009). Dimensionamento da equipe de enfermagem da UTI adulto de um hospital de ensino. Revista Eletrônica de Enfermagem, 11(1), 55-63.

Institute For Healthcare Improvemen. (2012). Prevent Ventilator- Associated Pneumonia. <http://www.ihi.org/resources/Pages/Too 1s/HowtoGuidePreventVAP.aspx>.

Lorente, L. Lecuona, M. Jiménez, A. Lorenzo, L. \& Roca, I. C, J.et al (2014). Continuous endotracheal tube cuff pressure control system protects against ventilator-associated pneumonia. Crit Care 21; 18 (2):R77.

Lourençone, E. M. S., et al (2019). Adesão às medidas preventivas versus incidência de pneumonia associada à ventilação mecânica. Revista de Epidemiologia e Controle de Infecção, 9(2): 142-148.

Mansano, F. P. N., et al (2017). Impacto de ação educativa na manutenção do decúbito elevado como medida preventiva de pneumonia associada à ventilação mecânica em Unidade de Terapia Intensiva. ABCS health sciences, 42(1): 21-26.

Maran, E. \& Spigolon, D. N. et al. (2019). Prevenção da pneumonia associada à ventilação mecânica sob a ótica de acadêmicos de enfermagem. Revista online de pesquisa cuidado é fundamental, 11(1): 132-137.

Melo, M. M., et al (2019). Pneumonia Associada à Ventilação Mecânica: Conhecimento dos Profissionais de Saúde Acerca da Prevenção e Medidas Educativas. Revista online de pesquisa cuidado é fundamental, 11(2)

Mendes, K. S., Silveira, R. C. C. P., \& Galvão, C. M. (2008). Revisão Integrativa: Método de pesquisa para a incorporação de evidências na saúde e na Enfermagem. Texto Contexto - Enfermagem, 17(4), 758- 64.

Rodrigues, A.N.; et al. (2016). Impactos e fatores determinantes no bundle de pneumonia associada à ventilação mecânica. Revista Brasileira de Enfermagem, 69(6), 1108-1114.

Rouzé, A., Jaillette, E., Poissy, J., Préau, S., \& Nseir, S. (2017). Tracheal Tube Design and Ventilator-Associated Pneumonia. Respiratory care, 62(10), 13161323. https://doi.org/10.4187/respcare.05492.

Santos, C. R., et al. (2018). Fatores de risco que favorecem a pneumonia associada à ventilação mecânica. Revista de Enfermagem UFPE on line, 12(12): 3401-3415.

Silva, L. D., Pereira, S. R. M., \& Mesquita, A. M. F. Procedimentos de enfermagem: Semiotécnica para o cuidado.

Sociedade Paulista de Infectologia (2006). Diretrizes sobre Pneumonia Associada à Ventilação Mecânica (PAV).

Taminato, M.; et al. (2019). Boas práticas na prevenção de pneumonia associada à ventilação mecânica. Acta Paulista de Enfermagem, 32 (1):11-17.

Wang, L. Li, X. Yang, Z. Tang, X. Yuan, Q. Deng, L. et al. (2016). Semi-recumbent position versus supine position for the prevention of ventilator-associated pneumonia in adults requiring mechanical ventilation. Cochrane Database Systemact Reviews. (1): CD009946.

Zanei, S. S. V. et al (2016). Valoração e registros sobre higiene oral de pacientes intubados nas unidades de terapia intensiva. Revista Mineira de enfermagem, 20: $[1-8]$.

Zigart, J. A. A. et al (2019). Adesão ao protocolo de pneumonia associado à ventilação mecânica. Revista de Enfermagem UFPE on line, 3(3): 655-663. 